\title{
Production Scheduling with Mould Maintenance in Flow Shop
}

\author{
Lu Shen ${ }^{1}$, Hongbing Yang ${ }^{1,2, a}$, Sheng Gao ${ }^{1}$ and Jia Fang ${ }^{1}$ \\ ${ }^{1}$ School of Mechanical and Electric Engineering, Soochow University, Suzhou 215021, China \\ ${ }^{2}$ Guangdong Provincial Key Laboratory of Computer Integrated Manufacturing, Guangdong \\ University of Technology, Guangzhou 510006, China \\ ayanghongbing@suda.edu.cn
}

Keywords: Mould maintenance, production scheduling, flow shop, genetic algorithm

Abstract. Unavailability of mould for preventive maintenance is usually ignored in research on production scheduling, this paper proposes to take setup and maintenance activities of mould into consideration and establishes an optimization model with objective of total weighted tardiness and earliness to demonstrate the impacts of mould on the production scheduling. Maintenance intervals assumed to be subject to shreshold with usage-based maintenance duration are jointly determined with sequencing problem as variables. Optimal solutions under different maintenance strategies are generated with genetic algorithm and comparative results illustrate the feasibility and effectiveness of the joint-scheduling under consideration of mould unavailability.

\section{Introduction}

Preventive maintenance is a recent extension of the scheduling area for resources were usually assumed to be available all the time. However, in most realistic situations, production schedules are often interrupted for resource unavailability like maintenance activities. With the realization of the interaction between production scheduling and maintenance planning, more and more attention has been drawn to the integration of both recently. Some researchers attempted to consider maintenance in various production systems with various maintenance strategies. Qi et al. ${ }^{[1]}$ considered machine's unavailability with preventive maintenance in a single-machine scheduling problem that proved to be NP-hard. Three heuristic algorithms and a branch-and-bound algorithm are proposed to minimize total completion time. Kenne et al. ${ }^{[2]}$ introduced an analytical model for the joint determination of age-dependent production planning and age-dependent PM to minimize an overall cost function. Berrichi et al. ${ }^{[3]}$ studied a scheduling problem with preventive maintenance to optimize makespan and system unavailability simultaneously. Two multi-objective genetic algorithms, NSGAII and WSGA (Weighted Sum Genetic Algorithm) are employed. Maintenance time was considered by Pan et al. ${ }^{[4]}$ as a variable subjected to machine degradation into single-machine scheduling problem to minimize the maximum weighted tardiness. Moradi et al. ${ }^{[5]}$ investigated flexible job shop problem with preventive maintenance activities that both the maintenance activity numbers and maintenance intervals are not fixed in advance. Four multi-objective optimization methods are compared to find the Pareto-optimal front in the flexible job-shop problem case. Wong et al. ${ }^{[6]}$ considered joint-scheduling with PM planning of mould to minimize the makespan in job shop plastic production systems. Mirabedini and Iranmanesh ${ }^{[7]}$ presented a new method of PM scheduling in two modes for more precise and better machine maintenance with serial jobs on parallel machines.

Researches on production scheduling with batch production like stamping system usually ignore the impacts of mould on the system much as it is among the most critical production resources despite the consideration of machine's unavailability. Mould plays an important role for the mix of 
sublots will cause frequent matching work which complicates schedules and affects delivery largely. In the literature of production scheduling, mould is usually assumed to be available through the whole process without consideration of the unavailability for setup and maintenance.

\section{Problem description}

The joint-scheduling model consists of $n$ types of products split into $L_{j}$ batches equally to be processed on $m$ machines in a flow shop. Each machine $k$ performs various products $j$ with specific mould $(j, k)$ and operating time $p t_{j k}$. With a particular mould loaded, setup time $M_{j k}$ needs considering if current sublot is different type with the former one. Besides, each mould is subject to the shreshold $M A$. Current age of mould is defined as the cumulated operated job quantity. In a real production system, maintenance can be implemented earlier with less maintenance time before reaching the shreshold while the periodic maintenance assumes maintenance occurs only once in a period before or when threshold.

Assumptions considered in this paper are summarized as follows:

(1) All items are available for processing at time zero; (2) No consideration about intermediate buffer is needed; (3) Setup times are sequence-independently included in processing times; (4) Sublot runs until completion once it has begun; (5) No interruption is allowed when a sublot is under production; (6) Intermingling of sublots between products of different types is allowed; (7) No quality issue is considered during scheduling.

Optimization model is formulated as belows:

$$
\begin{aligned}
& \min \sum_{j=1}^{n}\left\{\alpha_{j}\left(\max \left(\left(D_{j}-T_{j}\right), 0\right)\right)+\beta_{j}\left(\max \left(\left(T_{j}-D_{j}\right), 0\right)\right)\right\} \\
& T_{j} \geq C_{s j m}, s=1,2, \mathrm{~K} L_{j}, \\
& Q_{j}=\sum_{s=1}^{L_{j}} B_{j}, s=1,2, \mathrm{~K} L_{j}, j=1,2, \mathrm{~K} n, \\
& C_{s j k}-S T_{s j k}=p t_{j k} \times B_{j}, s=1,2, \mathrm{~K} L_{j}, j=1,2, \mathrm{~K} n, k=1,2, \mathrm{~K} m, \\
& C_{s j k}-p t_{j k} \times B_{j} \geq C_{(s-1) j k}, s=2, \mathrm{~L}, L_{j}, j=1,2, \mathrm{~K} n, k=1,2, \mathrm{~K} m, \\
& C_{s j k}-p t_{j k} \times B_{j} \geq C_{s j(k-1)}, k=2, \mathrm{~L}, m, s=1,2, \mathrm{~K} L_{j}, j=1,2, \mathrm{~K} m, \\
& S T_{s j k} \geq C_{(s-1)} m+t_{(s-1) j k}, s=2, \mathrm{~L}, L_{j}, j=1,2, \mathrm{~K} n, k=1,2, \mathrm{~K} m, \\
& a_{s j k}+B_{j} \times p t_{s j k} \leq M A, s=1,2, \mathrm{~K} L_{j}, j=1,2, \mathrm{~K} n, k=1,2, \mathrm{~K} m .
\end{aligned}
$$

Expression (1) describes the objective of minimizing the total weighted tardiness where $D_{j}$ is the demand of delivery and $T_{j}$ is defined in expression (2) as the completion time of product $j$ when its last sublot finishes the last process on machine $m$. Expression (3) makes sure that the total quantity $Q_{j}$ of product $j$ equals the sum of its sublots. Expression (4) defines that once a sublot starts, it will be finished without interruption, in other words, the difference between the completion time and starting time of a sublot is equal to its operation time. Expression (5), (6) makes sure that the processing of sublot $s$ of product $j$ starts only when its process on the previous machine is finished and previous lot on machine $k$ has left. Expression (8) is used to describe that maintenance must be implemented before reaching the shreshold $M A$. 


\section{Computational experiment}

In the numerical experiment, there are 10 hypothetical problems with various number of products and machines. Each sublot operates on every machine sequentially with specific mould. Parameters like the operation times, batch size and setup time are randomly generated. Maintenance intervals threshold for joint-scheduling is set as 5000 and maintenance durations are assumed to be linear with current age in Table 1 of moulds.

Table 1. Maintenance duration relative to current age

\begin{tabular}{cc}
\hline Current age of mould & Maintenance duration \\
\hline $0<a_{s j k}<=M A$ & 150 \\
$0.3^{*} M A<a_{s j k}<=0.7^{*} M A$ & $150+a_{s j k} / 20-75$ \\
$0.7^{*} M A<a_{s j k}<=M A$ & $300+a_{s j k} / 20+180$ \\
$M A<a_{s j k}$ & 720 \\
\hline
\end{tabular}

Genetic algorithm coded with MATLAB 7.8.0 (R2009a) on workstation Intel Core 2GB RAM and $2.4 \mathrm{GHz}$ processor is applied to solve the scheduling problem considering mould unavailability for setup and maintenance. The resulting formulation of joint-scheduling consists of the sequence of sublots and their maintenance variables in binary while that of periodic maintenance strategy only includes the first sequence part. The population size comprises 100 individuals. Crossover rate for genetic algorithm is 0.9 and mutation rate is 0.1 . The best, average and worst values of objective for above two maintenance strategies are obtained through 10 runs of genetic algorithm after 1000 iterations as shown in Table 2.

Table 2. Best, average and worst values of objective

\begin{tabular}{ccccccc}
\hline \multirow{2}{*}{ problem } & \multicolumn{3}{c}{ Peroidic } & \multicolumn{3}{c}{ Joint } \\
\cline { 2 - 6 } & Best & Avg. & Worst & Best & Avg. & Worst \\
\hline 1 & 1.11 & 2.50 & 3.58 & 0 & 0 & 0 \\
2 & 20.85 & 28.46 & 33.51 & 19,81 & 21.78 & 25.46 \\
3 & 8.78 & 13.99 & 18.34 & 7.74 & 10.43 & 12.69 \\
4 & 9.79 & 10.05 & 10.58 & 4.66 & 5.40 & 6.15 \\
5 & 1.31 & 1.93 & 3.09 & 0 & 0.53 & 3.06 \\
6 & 11.50 & 14.24 & 18.18 & 7.62 & 10.64 & 13.42 \\
7 & 5.03 & 7.59 & 10.02 & 0.43 & 1.87 & 2.76 \\
8 & 7.74 & 14.42 & 23.14 & 6.22 & 8.80 & 12.44 \\
9 & 12.23 & 16.81 & 21.85 & 4.77 & 11.44 & 15.15 \\
10 & 11.26 & 14.24 & 18.18 & 7.62 & 10.64 & 13.43 \\
\hline
\end{tabular}

As shown in Table 2, better results can be achieved in best, average and worst values for the objective of total weighted tardiness and earliness under each test problem with joint-scheduling strategy compared to periodic maintenance strategy with fixed maintenance duration. 


\section{Conclusions}

This paper presents a joint-scheduling model in flow shop to evaluate the interaction between production scheduling and mould unavailability in real manufacturing environment. Experiments with periodic and joint maintenance strategies are conducted with genetic algorithm and results show that joint-scheduling provides production planning more flexible schedules with a better balance between delivery and inventory. Further research will focus on integrating lot streaming with current model and more realistic parameters should be included such as other resource's unavailability. Taking those characteristics into consideration can promote effects and efficiency in production.

\section{Acknowledgements}

This work was financially supported by the Research Fund Program of Guangdong Provincial Key Laboratory of Computer Integrated Manufacturing (CIMSOF2016005) and the Undergraduate Innovational Experimentation Program of Soochow University (2016xj042).

\section{References}

[1] X. Qi, T. Chen and F. Tu: Journal of the Operational Research Society, Vol. 50 (1999), p. 1071-1078.

[2] J.P. Kenne, A. Gharbi and M. Beit: European Journal of Operational Research, Vol. 178 (2007), p. $408-420$.

[3] A. Berrichi, L. Amodeo, F. Yalaoui, E Chatelet and M Mezghiche: Journal of Intelligent Manufacturing, Vol. 20 (2008), p. 389-400.

[4] E. Pan, W. Liao and L. Xi: International Journal of Advanced Manufacturing Technology, Vol. 50 (2010), p. 365-375.

[5] E. Moradi, S.M.T.F. Ghomi and M. Zandieh: Expert Systems with Applications, Vol. 38 (2011), p. 7169-7178.

[6] C.S. Wong, F.T.S. Chan and S.H. Chung: International Journal of Production Research, Vol. 50 (2012), p. 5683-5697.

[7] S.N. Mirabedini and H. Iranmanesh: International Journal of Advanced Manufacturing Technology, Vol. 70 (2014), p. 1579-1589. 